\title{
The dynamic range extension system for the LHAASO-WCDA experiment
}

\author{
Cheng Liu*, ${ }^{*}$ Mingjun Chen, ${ }^{a}$ Xiaohan Ding, ${ }^{a b}$ Wenyan Du, ${ }^{a c}$ Bo Gao, ${ }^{a}$ Huihai He, ${ }^{a}$ \\ Huicai Li, ${ }^{a}$ Kai Li, ${ }^{a}$ Xiurong $\mathrm{Li},{ }^{a}$ Hanrong Wu, ${ }^{a}$ Zhiguo Yao ${ }^{a}$ for the LHAASO \\ collaboration \\ ${ }^{a}$ Institute of High Energy Physics, Chinese Academy of Sciences, Beijing, 100049, China \\ ${ }^{b}$ School of Physics, Liaoning University, Shenyang, 110000, China \\ ${ }^{c}$ Particle physics and nuclear physics major, Liaoning Normal University, Dalian, 116029, China \\ E-mail: Liucdihep.ac.cn
}

The Large High Altitude Air Shower Observatory (LHAASO) will be constructed at Mountain Haizi, in Sichuan Province, China (4410m a.s.l.). The Water Cherenkov Detector Array (WCDA), one of the major component of the LHAASO, will focus on surveying the northern sky for gamma ray sources from $100 \mathrm{GeV}$ to $30 \mathrm{TeV}$. The whole WCDA, owing an area of $78,000 \mathrm{~m}^{2}$, is subdividing into 3120 detector cells $\left(5 \times 5 \mathrm{~m}^{2}\right.$ each, with a water depth of $\left.4 \mathrm{~m}\right)$. Every cell has an 8 or 9-in PMT residing at the bottom to collect Cherenkov lights produced by the charged secondary particles of air showers. To observe high-energy shower events, especially to measure precisely the shower cores of events at the energy range from $100 \mathrm{TeV}$ to $10 \mathrm{PeV}$ for an analysis of the cosmic ray spectrum, as a dynamic range extension system, another sets of small PMTs (1 - 2 inch) will be added to 900 cells of the WCDA array. In this talk, the design and status of this dynamic range extension system is presented.

35th International Cosmic Ray Conference - ICRC2017

10-20 July, 2017

Bexco, Busan, Korea

${ }^{*}$ Speaker. 


\section{Introduction}

Cosmic rays (CRs) were discovered more than a century, however, the source of cosmic rays has remained mysterious. These high-energy particles observed at earth are mainly protons (89\%), but also nuclei of helium (10\%) and heavier nuclei (1\%). The all-particle energy spectrum of CRs is well described by a single power-law $\left(d N / d E \propto E^{\gamma}\right)$ over some 10 orders of magnitude variation in intensity. The spectral index is $\gamma \approx-2.7$ at energies up to several $\mathrm{PeV}$, then a steepening is observed, the so-called ąřkneeass, with $\gamma \approx-3.1$ at higher energies [四]. It is general believed that CRs below the knee originate from the Galaxy, however, the origin of the knee has not been well understood. The understanding of the knee is commonly thought to be a cornerstone for the understanding of the origin of (galactic) cosmic rays. The measurements of the energy spectrum of CRs around the knee region, in particular for individual nuclei, is an important tool to investigate the $\mathrm{CR}$ acceleration and propagation mechanism.

Accurate measurements of the showers in the energy region of the knees is one of the major motivations for the LHAASO experiment [ []]. The LHAASO is to be constructed at Mountain Haizi $\left(100.01^{0}\right.$ E, $29.35^{\circ} \mathrm{N} ; 4410 \mathrm{~m}$ above sea level) in Sichuan Province, China. It consists of an area of $1 \mathrm{~km}^{2}$ array (KM2A), water Cherenkov detector array (WCDA), and wide field-of-view imaging Cherenkov telescope array (WFCTA), forming as a hybrid extensive air shower (EAS) array. The KM2A is comprised of 5195 electromagnetic particle detectors (EDs) with $1 \mathrm{~m}^{2}$ each and a spacing of $15 \mathrm{~m}$, and 1171 muon detectors (MDs) with $36 \mathrm{~m}^{2}$ each and a spacing of $30 \mathrm{~m}$. The WCDA, covering an area $78000 \mathrm{~m}^{2}$ and subdividing into 3120 detector cells, will be built at the center of KM2A. Atmosphere above the LHAASO-WCDA is watched by the WFCTA, which is made up of 12 telescopes.

Using all detector arrays in LHAASO, one is able to measure an air shower with multiple relevant parameters, such as the shower geometry by WCDA, shower energy by WFCT and muon content outside the core region of the shower by MDs. The dynamic range extension system of the LHAASO-WCDA is to precisely measure the shower cores of events at the energy range from $100 \mathrm{TeV}$ to $10 \mathrm{PeV}$. Combining the shower cores information, those parameters will be useful to identify the composition of the primary particles with high purity. Details of this dynamic range extension system of the LHAASO-WCDA are described below.

\section{LHAASO-WCDA Array}

The LHAASO-WCDA consists of three pools, two of which will cover areas of $150 \mathrm{~m} \times 150 \mathrm{~m}$, and the other area of $300 m \times 110 m$ (see Fig. W). Each pool is subdivided into cells with an area of $5 m \times 5 m$, separated by black plastic curtains to prevent the cross-talk of light between cells. Every cell has an 8 or 9-in PMT resided at the bottom and facing upwards with an effective water depth of $4 \mathrm{~m}$ above the photo-cathode.

When a shower particle goes through a detector cell, it will induce cascade processes which produce Cherenkov light in water. Some of the light may arrive at the photo-cathode of the PMTs. The light intensity is proportional to the total energy carried by the air shower charged particles and photons in the cell. The energy flux, measured by the total number of equivalent PEs on PMTs, in the cell hit by the core mainly carried by secondary particles produced in last few generations of 


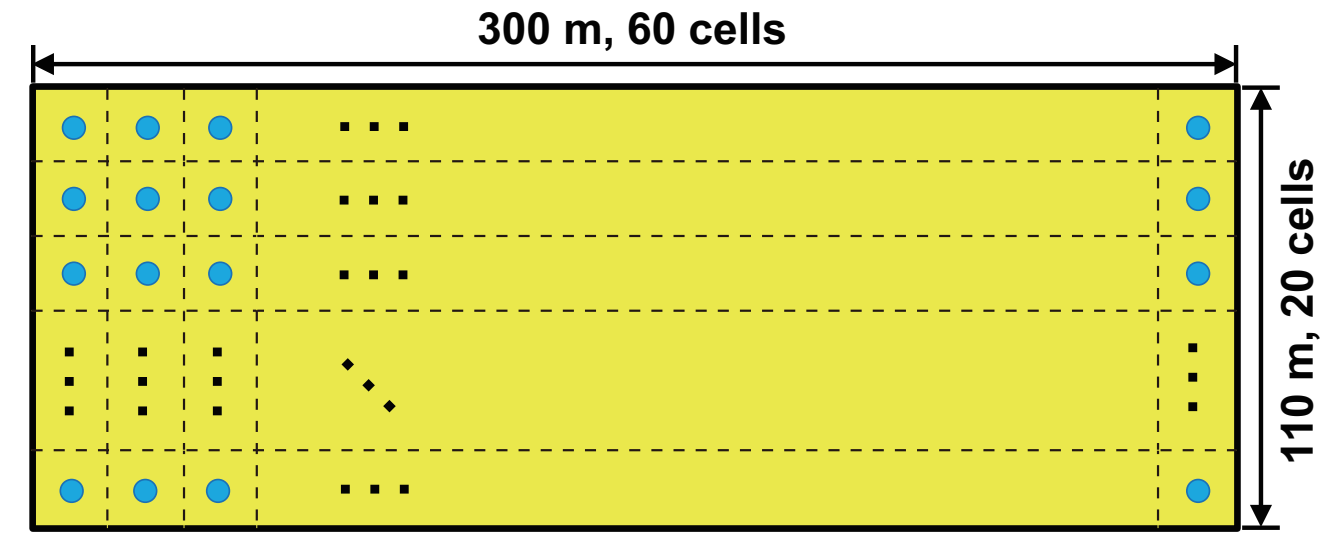

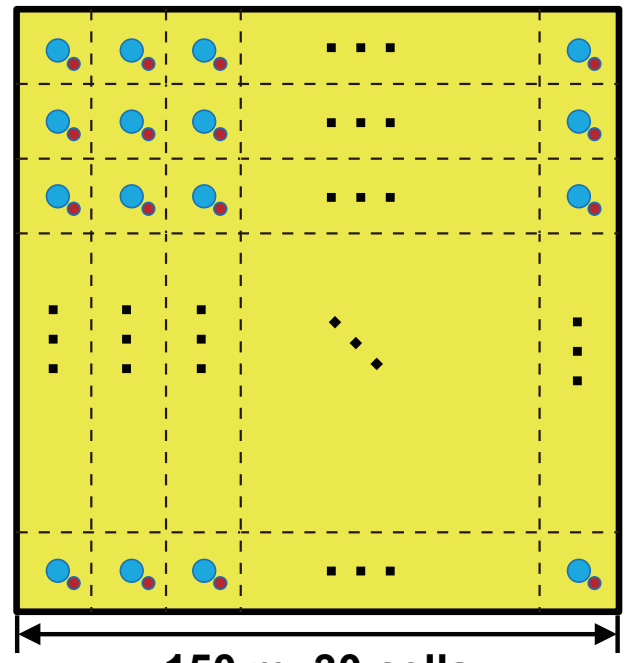

$150 \mathrm{~m}, 30$ cells

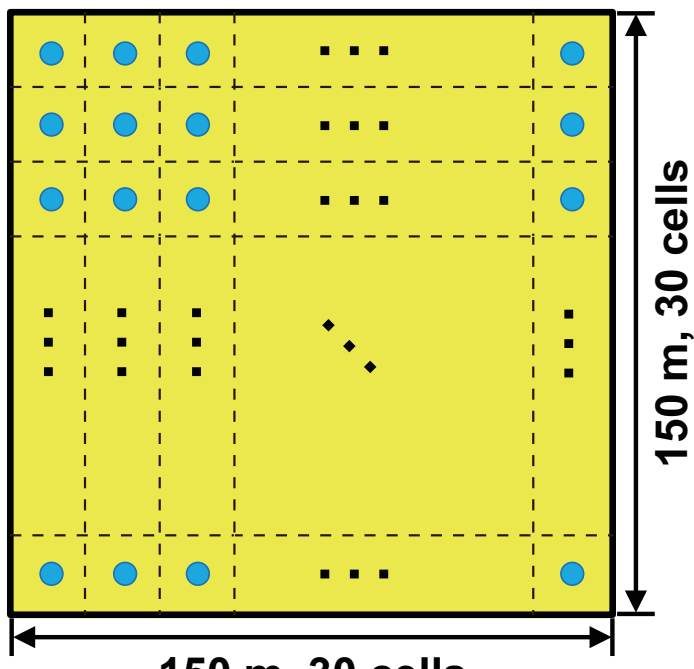

$150 \mathrm{~m}, 30$ cells

Figure 1: The schematic of the LHAASO-WCDA layout.

the air shower. These particles, without suffering many Coulomb scattering, is a good measure of the hadrons produced in the last few generations. It's a useful parameter sensitive to the identity of the primary particle, because the number of hadrons in a shower reduces with the elongation of the shower quite sensitively [B]].

To measure precisely the shower cores of events at the energy range from $100 \mathrm{TeV}$ to 10 PeV by LHAASO-WCDA, 900 cells of the WCDA array are planned to add another $1-2$ inch PMT at side of the big PMT, as a dynamic range extension system. An example of using particle density near the shower core and the shower Cherenkov image shape, has been analyzed on the data produced by the combined experiment with ARGO-YBJ and a prototype telescope of LHAASOWFCTA. It has reported an important discovery of the knee of the spectrum (a bending of the $\mathrm{p} / \mathrm{He}$ spectrum below $10^{15} \mathrm{eV}$, at about $700 \mathrm{TeV}$ ). The LHAASO, has 4 different kinds of sub-detectors, will enable the measurements with greatly improved performance. Monte Carlo (MC) simulation shows that, using all detector arrays in LHAASO, for a mixed sample of proton plus Helium nuclei can reach an even higher purity such as $95 \%$ [四]. In this talk, the design and status of this dynamic range extension system is described. 


\section{Design of the dynamic range extension system}

The dynamic range of the 8 or 9-in PMTs (large PMTs) is designed from 1 photoelectron to 4000 PEs to survey the VHE gamma ray sources (from $100 \mathrm{GeV}$ to $30 \mathrm{TeV}$ ). For a $100 \mathrm{TeV}$ primary protons, simulation shows that, the average number of photoelectrons recorded by large PMT near the shower core is above 30000 [[]]. To achieve the dynamic range expansion, experiments require a smaller PMT. For example, compared to an 8 inch PMT, the 1 inch PMT will have a 64 fold reduction in the receiving area. MC simulation shows that, the dynamic range for small PMTs is required from 50 PEs to 500,000 PEs. In this talk, a 1.5 inch PMT of CR285 is selected as a candidate of LHAASO-WCDA.

\subsection{Waterproof design}

A typical 1.5 inch PMT is a cylinder with a length of only $10 \mathrm{~cm}$. Compared with large PMTs, its packing area is much smaller. At present, we are investigating two package designs for small PMTs. One solution is to package glass surfaces of the PMT by using O-rings as shown in Fig. I(a). In this way, the photocathode of PMTs can be used to directly measure the Cherenkov light produced in the water. The other solution is to put the PMT in a closed box and a glass sheet is enclosed at the top of the box, as shown in Fig. $\mathbf{Z}(\mathrm{b})$. Because the small PMTs are used to measure the large signals, the absorption effect of glass on photons can be neglected. This method does not operate on the PMT, and the design is simple. The estimate of these two plans is still in progress, and the final plan has not been determined.
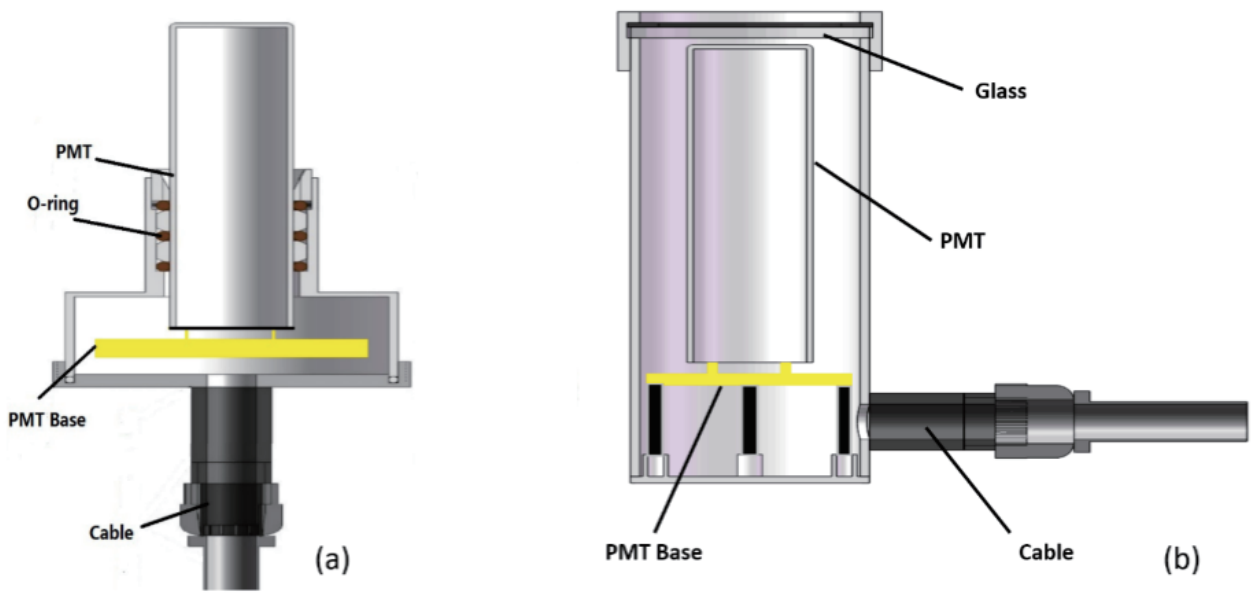

Figure 2: The waterproof designs for small PMTs.

\subsection{The dynamic range base design}

The PMT exhibits a good linearity in anode output current over a wide range of incident light levels as well as the photon counting region. However, if the incident light amount is too large, confined by the space charge effects, the output current begins to deviate from the ideal linearity. In our case, the double readouts for dynode and anode are adopted to broaden the dynamic range. CR285 has 10 dynodes and the 7th is selected to be an output dynode. Fig.B shows the schematic 
of the high dynamic base circuit. It is based on a passive resistor chain. The voltage distribution on the electrodes of the PMT is tapered.

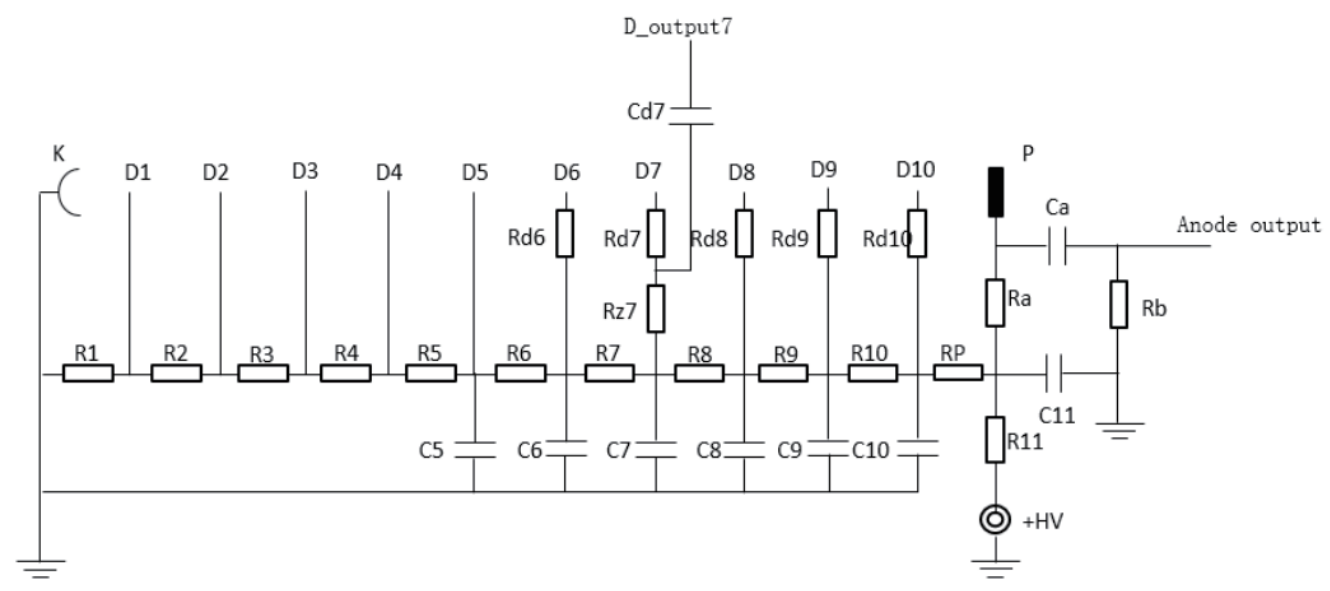

Figure 3: Schematic diagram of voltage-divider circuit for PMT CR285.

According to the design of figure 3, the dynamic range of the anode output is 50-10,000 PEs, while the dynode output covers the dynamic range from 2500 to 500,000 PEs. The overlap region can be used to calibrate the gain of the dynode output. The charge non-linearity of the anode and the DY7 are shown in Fig.t. The results show that, applying the high dynamic range base circuit, the requirements of the 4 orders of dynamic range can be satisfied.

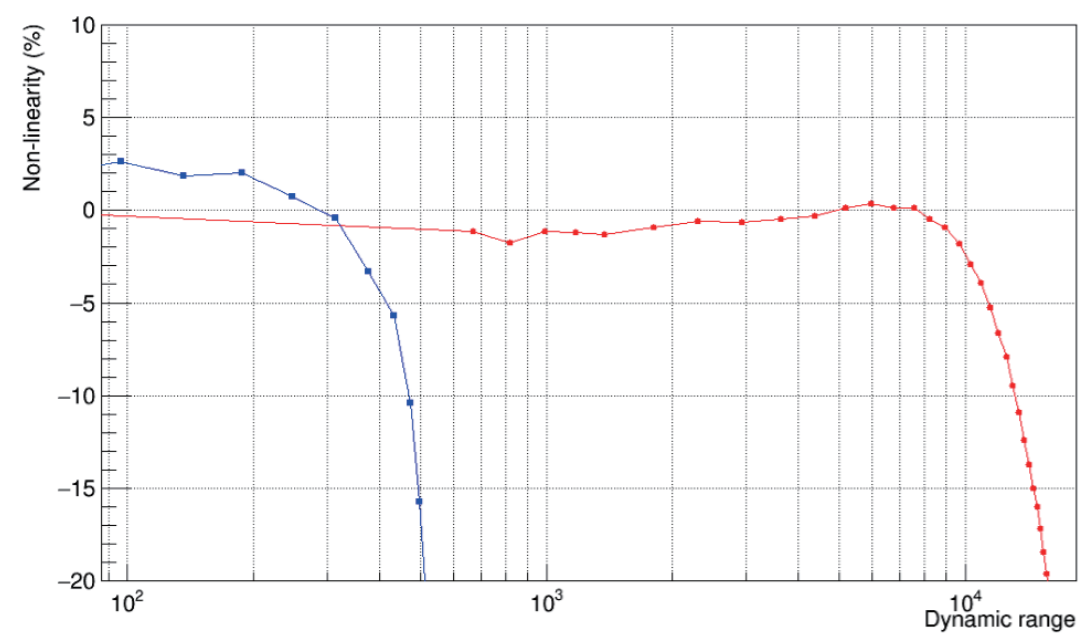

Figure 4: The charge non-linearity of the anode and DY7.

\subsection{Readout electronics}

To achieve such a large scope as mentioned above and enough overlap range, two electronics channels and two signals readout channels are employed. Signals from the anode and dynode 
are digitized by a two-channel ADC (Analog to Digital Converter) after being manipulated by the amplification and shaping circuit. Then, the digitized signals are sent to the FPGA for digital peaking. The architecture of the readout electronics for charge digitizing module is designed as shown in Fig. [.].

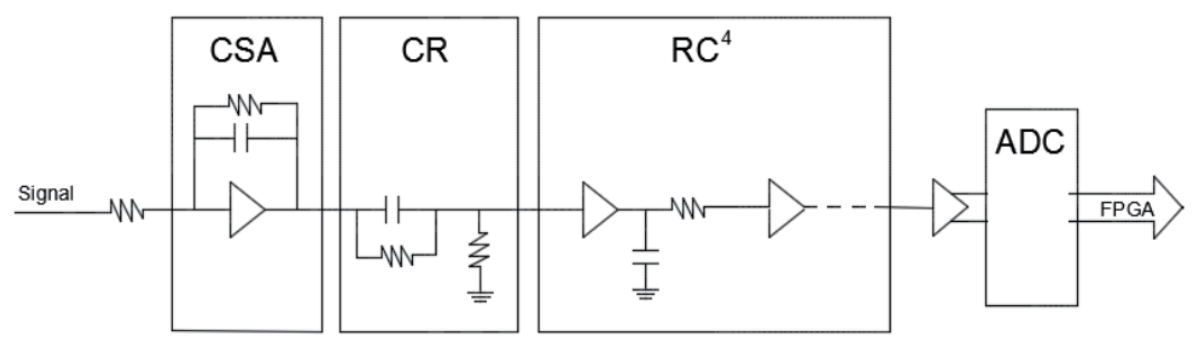

Figure 5: Design of charge digitizing module for readout electronics.

According to the event rate of CRs above $100 \mathrm{TeV}$, the single counting rate of each cell is about $10 \mathrm{~Hz}$. The time digitizing precision is less than $20 \mathrm{~ns}$, which is no problem with match the large PMTs single. All digitized data is transmitted to DAQ (Data Acquisition) via a simplified White Rabbit protocol.

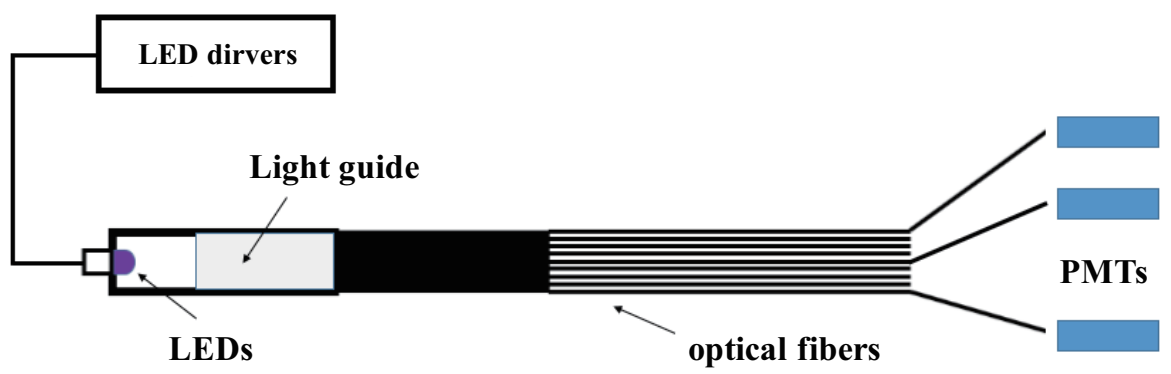

Figure 6: Schematic of charge calibration for small PMTs.

\subsection{Charge calibration}

During the operation of the small PMTs, to measure precisely the shower cores of events, a charge calibration system based on an array of LEDs and plastic optical fiber bundle is designed as shown in Fig. 6. Combined with the layout of the electronics system, the design of the calibration system is also based on the concept of "cluster", which contains $6 \times 6$ cells. The LEDs, controlled via individual drivers, can be turned on individually or in any combination of the array. Using the different light emitted by the LEDs array, we can measured the SPE spectrum and the high voltage response of each PMT. A linear fit of $\log 10$ (gain) versus $\log 10$ (highvoltage) allows the high voltage of each PMT to be tuned to the desired operating point $\left(2 \times 10^{5}\right.$ for small PMTs). 


\section{Summary}

To observe high-energy shower events, especially to precisely measure the shower cores of events at the energy range from $100 \mathrm{TeV}$ to $10 \mathrm{PeV}$ for an analysis of the cosmic ray spectrum by LHAASO, 900 cells of the WCDA array are planned to add another 1 - 2 inch PMT at side of the big PMT, as a dynamic range extension system. In this talk, we have described package designs of the small PMTs, the dynamic range base design and the readout electronics system. Using all detector arrays in LHAASO, combining the shower cores information, it will be very powerful to identify the composition of the primary particles with high purity.

\section{Acknowledgements}

This work is supported by National Natural Science Foundation (NSFC) of China under contacts No. 11635011, 11405180, 11405181.

\section{References}

[1] Blumer, J., Engel, R., \& Horandel, Jorg R., Progress in Particle and Nuclear Physics, 2009, 63, 293

[2] Cao, Z., Chinese Phys. C, 2010, 34, 249

[3] L. Ding, X. Lan, Y. Hou, X. Zhou, H. Jia, Z. Cao, Nucl.Phys.Proc.Suppl., 2008, 162, 175-176

[4] B. Bi, Z. Cao, Y. Liu, et al., Frascati Physics Series Vol., 2016, 64

[5] Xiurong Li et al., PoS(ICRC2017) 